\title{
Segregation in Swarms of Mobile Robots Based on the Brazil Nut Effect
}

\author{
Roderich Groß, Stéphane Magnenat, and Francesco Mondada
}

\begin{abstract}
We study a simple algorithm inspired by the Brazil nut effect for achieving segregation in a swarm of mobile robots. The algorithm lets each robot mimic a particle of a certain size and broadcast this information locally. The motion of each particle is controlled by three reactive behaviors: random walk, taxis, and repulsion by other particles. The segregation task requires the swarm to self-organize into a spatial arrangement in which the robots are ranked by particle size (e.g., annular structures or stripes).

Using a physics-based computer simulation, we study the segregation performance of swarms of 50 mobile robots. The robots represent particles of three different sizes. We first analyze the problem of how to combine the basic behaviors so as to minimize the percentage of errors in rank. We then show that the system is very robust to noise on inter-robot perception and communication. For a noise level of $50 \%$, the mean percentage of errors in rank is $1 \%$. Moreover, we investigate a simplified version of the control algorithm, which does not rely on communication.
\end{abstract}

Finally, we show that the mean percentage of errors in rank decreases exponentially as the particles' size ratio increases. As the error is bounded, one can achieve $100 \%$ error-free segregation. The reduction in error, however, comes at the expense of an increase in the required sensing/communication range.

Index Terms-Annulus, Brazil nut effect, center-periphery, muesli effect, pattern formation, robots, segregation, sorting, stripes, swarm intelligence

\section{INTRODUCTION}

One of the grand challenges in engineering is the design of decentralized systems composed of numerous autonomous embodied agents for applications at scales from the macro to the nano. Nature offers a wide range of decentralized systems in which collective phenomena emerge as a result of a selforganized process [1]. In the last decades, researchers of various disciplines have identified some of the underlying principles of such decentralized and self-organizing natural systems and transferred them to technology [2], [3], [4].

One example of a natural collective phenomenon is the coordinated motion in groups of animals, such as a flock of birds, a school of fish, or a crowd of humans. Inspired by this phenomenon, Reynolds [5] created flocks of artificial agents in a computer simulation. He identified a set of three

R. Groß acknowledges support from the Sixth Framework Programme of the European Community in the form of a Marie Curie Intra-European Fellowship (contract no. MEIF-CT-2006-040312). The work reflects only the authors' views. The European Community is not liable for any use that may be made of the information.

R. Groß, S. Magnenat, and F. Mondada are with the Robotic Systems Laboratory (LSRO), Ecole Polytechnique Fédérale de Lausanne, Station 9, 1015 Lausanne, Switzerland roderich dot gross at ieee dot org, stephane at magnenat dot net, francesco dot mondada at epfl dot ch

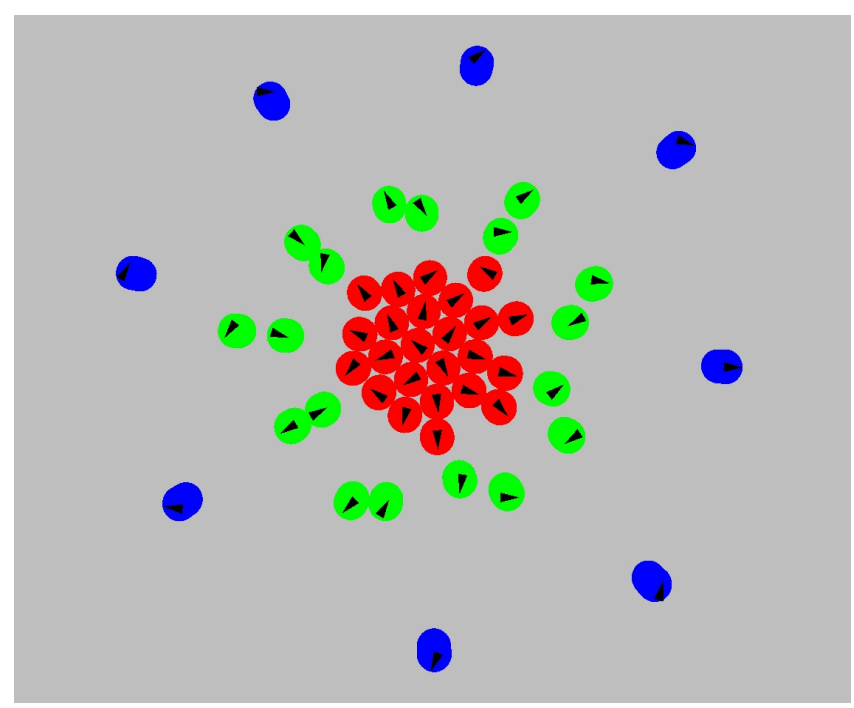

Fig. 1. Segregation in a swarm of 50 mobile robots (top view). Each robot mimics a particle of a certain size. Red, green, and blue robots mimic particles of radius $6 \mathrm{~cm}, 12 \mathrm{~cm}$, and $24 \mathrm{~cm}$, respectively. The robot's motion is governed by three behaviors: a vector pointing in a random direction, a vector pointing towards the center (not indicated), and vectors pointing away from any intersecting particles. The combination of these vectors leads to the formation of an annular structure.

simple rules that generated flocking behaviors. The first rule let agents avoid colliding with each other. The second rule enabled nearby agents to match their headings and speed. The third rule enabled agents to stay close to nearby flock mates. The rules produced three vectors which were then combined in a way to produce the overall behavior.

In this paper, we take inspiration from the collective phenomenon that segregation occurs when shaking constantly a mixture of particles of different sizes [6]. The underlying mechanism is known as the Brazil nut effect or the muesli effect. Barker and Grimson [7] describe it as follows: "During the periods when shaking loosens the packing, individual small particles can move into voids beneath large particles and so prevent them from returning to their previous positions. It is far less probable that several small particles will move together so as to create a void that can be occupied by a single large particle. The net effect is that the smaller particles occupy the lower positions during the active part of the shaking process and then become trapped there when the grains fix into a new arrangement." As reported in [8] the diameter ratio of the different particles is a critical variableincreasing this ratio leads to a decrease in the segregation error. 
To understand segregation phenomena is of vital interest to the food processing industries, involving powders and other foods made of particles or granules. Our primary motivation however is to develop a simplistic control algorithm that allows mobile robots to form 2-D or 3-D ordered patterns, such as the annular structure shown in Fig. 1. Potential application domains of such structures (including centerperiphery formations) comprise construction, encapsulation and defense strategies.

Melhuish et al. [9], [10] and Wilson et al. [11] investigated simple rules to let a colony of physical robots built annular structures by pushing and pulling pucks of particular size. They explored several mechanisms inspired by the behavior of Leptothorax ants [12]. One mechanism was based on the Brazil nut effect, that is, to promote segregation based on differences in the pucks' sizes. Another mechanism let the robots introduce an object specific spacing before dropping the object. The robots formed structures of pucks showing segregation, though the performance was not optimal (regardless of the mechanism used). One reason for this was that multiple performance criteria were targeted: In addition to segregation, the compactness, shape, and completeness of the resulting structure were considered. Moreover, Wilson et al. [11] state "there is [...] no force similar to a gravitational force, required for muesli sorting, toward the centre of the cluster. Here gravity is replaced by a clustering tendency provided by the robots, but this does not seem to provide the objects with a strong enough pull towards the centre of the cluster."

Different from the studies of Melhuish et al. and Wilson et al. our robots simulate a particle system under vibration. We thus directly exploit the Brazil nut effect. The robots represent the particles themselves. The particle size however exceeds the robot's physical dimensions. All robots (and all particles) are continuously in motion.

We first analyze the system under the assumption that the robots' sensors are not affected by noise. In fact, the particular level of noise would likely depend on the technology used (see Section IV). We perform a systematic study to choose a good combination for the algorithm's most intrinsic parameters. We then analyze the time dynamics of the segregation process. Moreover, we examine the performance of the system for different levels of noise. Based on the knowledge gained, we propose a simplified version of the control algorithm, which does not rely anymore on interrobot communication. Finally, we show that the system is capable of error-free segregation (provided the sensing range is large enough).

\section{Methods}

\section{A. Simulation model}

The simulator models the kinematics and dynamics of rigid bodies in two dimensions (2-D) using the open-source Enki simulation toolkit [13]. Enki provides a faster than realtime simulation of groups of mobile robots that move on flat terrain. It has built-in support for several existing robotic

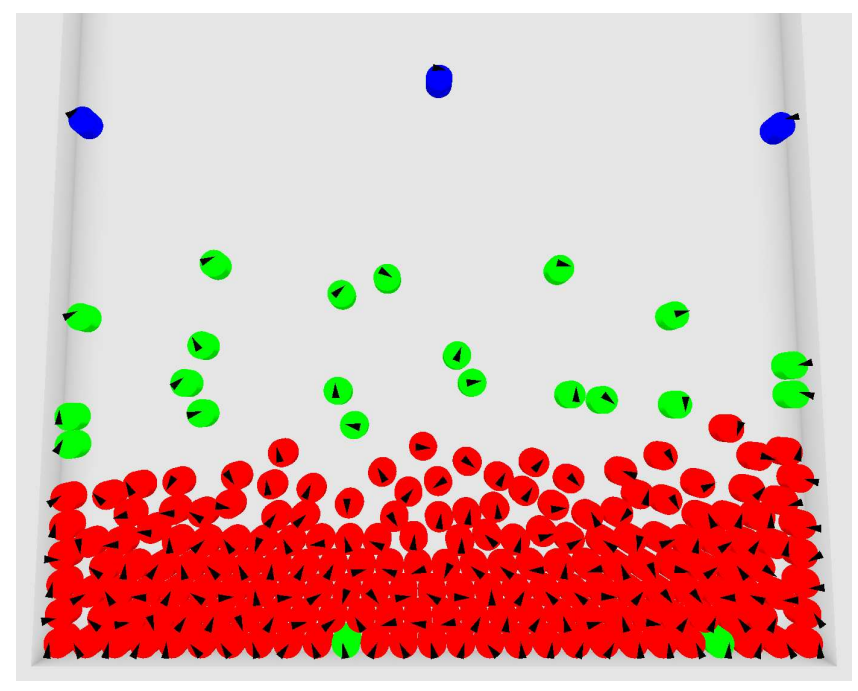

Fig. 2. Stripe pattern formed by a swarm of 200 robots in a bounded environment.

platforms. Enki can also be used by the Webots ${ }^{\mathrm{TM}}$ simulator in 2-D mode [14].

The 2-D space is modeled continuously (in double precision). Time progresses in discrete steps of $1 / 30 \mathrm{~s}$. The length of the control cycle is $\Delta=0.1 \mathrm{~s}$.

The core characteristics of the robot model are those of the e-puck robot [15]. It weighs $152 \mathrm{~g}$. Its body is modeled as a disk of radius $3.7 \mathrm{~cm}$. Two wheels are mounted symmetrically on a single axis crossing the center (with an inter-wheel distance of $5.1 \mathrm{~cm}$ ). Each wheel can be controlled independently by setting a speed (in $\mathrm{cm} / \mathrm{s}$ ) within the range $[-M, M]$, where $M=12.8$. The speed of each wheel is subjected to $\pm 5 \%$ uniform noise, which is updated at every control cycle.

We assume that robot $i, i=1, \ldots, n$, can identify the angular position $\alpha_{i}$ of a predefined destination (in the coordinate system of robot $i$ ). In our study, we refer to the destination as the "center" of the environment. The robots are expected to form patterns of annuli around this center, as those shown in Fig. 1. Note that in a bounded environment, the destination could be outside the boundaries. The robots could then form a stripe pattern as shown in Fig. 2.

Each robot broadcasts a simple signal that can be received by other robots in its vicinity. Robot $i$ receives the signal emitted by robot $j \neq i$ if and only if the distance between the two (from center to center) does not exceed range $R$. In our particular study, the range is $R=48 \mathrm{~cm}$. As explained in Section II-B, any larger range would produce the same system behavior. For any robot $j$ within range $R$, robot $i$ receives the corresponding signal and can estimate the distance $\left(d_{i, j}\right)$ to and the angular position $\left(\gamma_{i, j}\right)$ of the emitter (in the coordinate system of robot $i$ ). These features are not directly available on the e-puck robot, but are supported by several e-puck module extensions (see Section IV).

We start with the assumption that each robot receives correctly the signal from any nearby robot, and that it can calculate the exact position of the emitter $\left(d_{i, j}, \gamma_{i, j}\right)$. This 
assumption will be relaxed in Sections III-C and III-D.

For the generation of pseudo-random numbers, we use the 2002 revision of the MT19937 generator [16].

\section{B. Controller}

We consider robot $i, i=1,2, \ldots, n$, as a disk of radius $r_{i}$. The disk is only a control concept and does not reflect the robot's physical dimensions. However, it is assumed that

$$
\begin{aligned}
\min _{i} r_{i} & \geq r, \\
\max _{i} r_{i} & \leq \frac{R}{2},
\end{aligned}
$$

where $r$ is the radius of the smallest circle that can fully contain the robot, and $R$ is the robots' sensing range (see Section II-A). We assume that each robot can broadcast locally information that enables other robots to identify the size of its disk. This assumption can be relaxed as explained later.

Each robot executes the same control algorithm. The algorithm is implemented using the motor schema paradigm [17]. At the beginning of each control cycle (i.e., every $\Delta=$ $0.1 \mathrm{~s}$ ), robot $i$ generates schema instantiations for three motor schemas:

- $\boldsymbol{v}_{i}^{\text {(taxis) }}$, a unit vector pointing towards a predefined destination. Formally,

$$
\boldsymbol{v}_{i}^{\text {(taxis) }}=\left(\begin{array}{c}
-\sin \alpha_{i} \\
\cos \alpha_{i}
\end{array}\right),
$$

where $\alpha_{i}$ is the angular position of the destination (see Section II-A). In our particular study, the destination is referred to as the center of the environment.

- $\boldsymbol{v}_{i}^{\text {(rand) }}$, a unit vector pointing in a random direction. Formally,

$$
\boldsymbol{v}_{i}^{(\text {rand })}=\left(\begin{array}{c}
-\sin \beta_{i} \\
\cos \beta_{i}
\end{array}\right),
$$

where $\beta_{i}$ is uniformly sampled from $[0,2 \pi)$. At the beginning of each control cycle, $\beta_{i}$ is updated with probability $1 / \Delta$.

- $\boldsymbol{v}_{i}^{(\mathrm{repul})}$, a vector that represents the cumulative repulsion force by other nearby robots. Robot $i$ is repelled by robot $j \neq i$ if and only if the disks of robots $i$ and $j$ overlap. Formally,

$$
\begin{aligned}
& \boldsymbol{v}_{i}^{(\text {repul })}=\sum_{j \neq i} \boldsymbol{v}_{i, j}^{(\text {repul })} \\
& \boldsymbol{v}_{i, j}^{\text {(repul) }}= \begin{cases}\left(\begin{array}{c}
\sin \gamma_{i, j} \\
-\cos \gamma_{i, j}
\end{array}\right) & d_{i, j}<r_{i}+r_{j} ; \\
\mathbf{0} & \text { otherwise },\end{cases}
\end{aligned}
$$

where $\gamma_{i, j}$ and $d_{i, j}$ are respectively the angular position and the distance of robot $j$. Note that $\boldsymbol{v}_{i}^{\text {(repul) }}$ can be computed based on local information, as robot $i$ can only be repelled by robot $j$ if the latter is within range $R$ [see Equation (2)]:

$$
r_{i}+r_{j} \leq 2 \max _{k} r_{k} \leq R
$$

The three motor schema instantiations are combined as follows:

$$
\boldsymbol{v}_{i}=\boldsymbol{v}_{i}^{\text {(taxis) }}+c^{(\text {rand) }} \boldsymbol{v}_{i}^{(\text {rand })}+f\left(c^{(\text {repul) }} \boldsymbol{v}_{i}^{(\text {repul) }}\right)
$$

Parameters $c^{(\text {rand) }}$ and $c^{(\text {repul) }}$ are used to weight the relative impact of the motor schema instantiations. Function $f(\cdot)$ adjusts the norm of the weighted repulsion vector to $M / 2$ in case the norm exceeds this limit. Whereas the other two motor schema instantiations are unit vectors, the norm of $\boldsymbol{v}_{i}$ depends on the number, type, and arrangement of other disks. Function $f(\cdot)$ thus limits the impact of repulsions.

The speed of the left and right wheels, $s_{i, l}$ and $s_{i, r}$, are calculated as follows:

$$
\left(\begin{array}{c}
s_{i, l} \\
s_{i, r}
\end{array}\right)=\left(\begin{array}{cc}
1 & 1 \\
-1 & 1
\end{array}\right) \boldsymbol{v}_{i} .
$$

If $s_{i, l}$ (or $s_{i, r}$ ) is not within range $[-M, M]$, it is clamped to the corresponding bound.

\section{Experimental setup}

We consider a system with $n=50$ robots and with $m=3$ different sizes of disks. Thus there are three homogeneous groups of robots to be segregated. The radius of the $i$ th smallest disk, $r^{(i)}$, is chosen as follows:

$$
r^{(i)}=a b^{i-1},
$$

where $a$ is the size (in $\mathrm{cm}$ ) of the smallest disk and $b$ is the minimum size ratio between disks of different groups. We use $a=6 \mathrm{~cm}$ and $b=2$. From Equation (7), it follows that $R$ must be at least $2 \max _{i} r_{i}=2 a b^{m-1}=48 \mathrm{~cm}$.

Ideally, we expect the robots to organize into the following spatial arrangement: The disks of radius $r^{(i)}(i=$ $1,2, \ldots, m)$ are fully contained within the area of the annulus formed by the concentric circles of radii $(i-1) g$ and $i g$ in the center of the environment. Parameter $g$ represents the "thickness" of the annulus. Consequently, the disk of each robot is fully contained in the area represented by a disk of radius $m g$ in the center of the environment.

To obtain the ideal pattern, we must choose $n$ as well as $n^{(i)}$, the number of robots with disk radius $r^{(i)}$. Variable $n^{(i)}$ is set proportional to the ratio of the area of the corresponding annulus [i.e., $\left.\pi g^{2}\left(i^{2}-(i-1)^{2}\right)\right]$ and the area of each disk [i.e., $\pi\left(r^{(i)}\right)^{2}$ ]. Setting $n=n^{(1)}+n^{(2)}+\cdots+n^{(m)}$ and eliminating variable $g$, one obtains:

$$
n^{(i)}=\frac{\frac{2 i-1}{\left(r^{(i)}\right)^{2}}}{\sum_{j=1}^{m} \frac{2 j-1}{\left(r^{(j)}\right)^{2}}} n .
$$

To achieve the annuli's thickness $g$ and thus the pattern's overall dimensions, the number of robots $n$ can be chosen as follows:

$$
n=g^{2} \sum_{j=1}^{m} \frac{2 j-1}{\left(r^{(j)}\right)^{2}} .
$$

It is worth noting that we do not aim at forming the exact pattern as described above. The calculated numbers of disks 

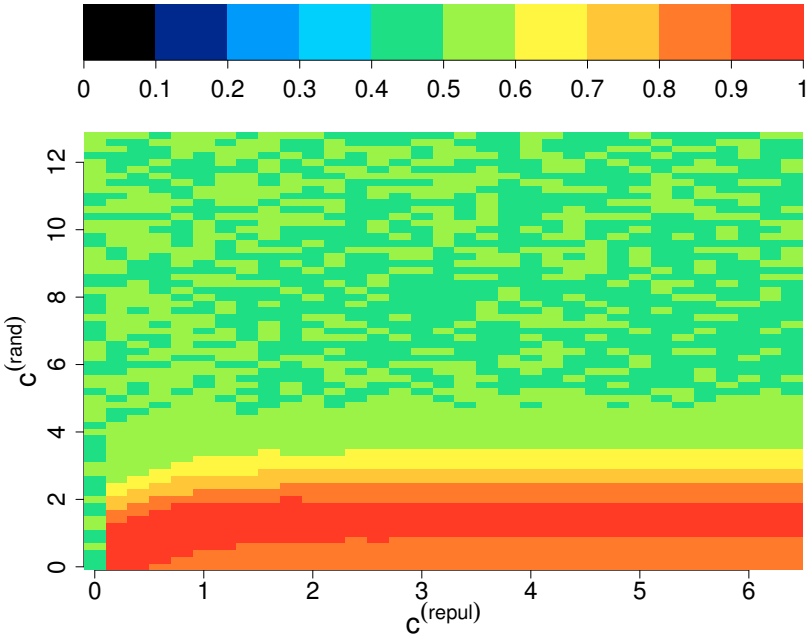

Fig. 3. Mean segregation error for samples taken over the entire parameter range (linear scale, 100 trials per parameter combination).

are approximate only because the packing density of disks depends on the disks' radii.

At the beginning of a simulation trial, $n$ robots are placed, one after the other, at non-overlapping random positions and with random orientations in a circular area in the center of the environment. This area corresponds to an (initial) density of $200 \mathrm{~cm}^{2}$ per robot. Subsequently, the robots are selected for the $m$ groups of different disk radii. To avoid any bias, the robots are selected at random and independently from the order in which they were assigned to their positions. The required number of robots with disk radii $r^{(i)}$ is obtained from Equation (11). In principle, each robot could choose disk radius $r^{(i)}$ with probability $\frac{n^{(i)}}{n}$. Note that this probability does not depend on $n$.

\section{Performance metric}

We consider two robots $i$ and $j$ at absolute positions $\boldsymbol{x}_{i}$ and $\boldsymbol{x}_{j}$. Let $\boldsymbol{o}$ denote the destination-in this study the center of the environment. The segregation error of robot pair $(i, j)$ is defined as

$$
e_{i, j}= \begin{cases}1 & \left(r_{i}<r_{j}\right) \wedge\left(\left\|\boldsymbol{x}_{\boldsymbol{i}}-\boldsymbol{o}\right\| \geq\left\|\boldsymbol{x}_{\boldsymbol{j}}-\boldsymbol{o}\right\|\right) ; \\ 1 & \left(r_{i}>r_{j}\right) \wedge\left(\left\|\boldsymbol{x}_{\boldsymbol{i}}-\boldsymbol{o}\right\| \leq\left\|\boldsymbol{x}_{\boldsymbol{j}}-\boldsymbol{o}\right\|\right) ; \\ 0 & \text { otherwise. }\end{cases}
$$

The segregation error for the entire swarm is obtained by summing up the segregation errors for all possible pairs of robots. Thereby, the metric evaluates the pattern based on all possible errors in the robots' rank.

The segregation error is scaled such that 0 and 1 correspond respectively to the best possible and least possible segregation errors. A segregation error of 0.5 corresponds to placements without positional bias. For example, the expected segregation error of robots in their initial placement is 0.5. A segregation error of 1 can only be obtained if the disks are ranked in the exact opposite order.
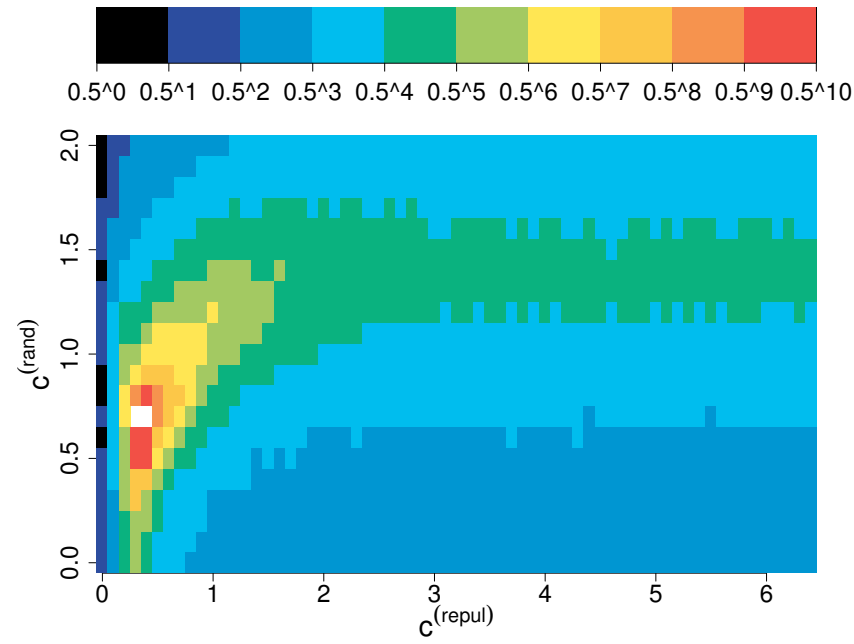

Fig. 4. Mean segregation error for samples taken over a focused parameter range (log-scale, 100 trials per parameter combination).

\section{RESULTS}

\section{A. The influence of control parameters $c^{(\text {(rand) })}$ and $c^{(\text {repul })}$}

In the following, we investigate the performance of a swarm of $n=50$ robots that consists of $m=3$ homogeneous groups: According to Equation (11), the swarm consists of 24 robots representing disks of radius $6 \mathrm{~cm}, 18$ robots representing disks of radius $12 \mathrm{~cm}$, and 8 robots representing disks of radius $24 \mathrm{~cm}$ (see also Fig. 1).

We took samples over the whole range of control parameters: $c^{(\text {rand })} \in\{0,0.2,0.4,0.6, \ldots, M\}$ and $c^{(\text {repul })} \in$ $\{0,0.2,0.4,0.6, \ldots, M / 2\}$. For each parameter combination we conducted 100 independent simulation runs (i.e., 214500 independent simulation runs in total). The simulation period was $600 \mathrm{~s}$. The segregation error was computed at the end of each simulation run.

Fig. 3 shows the mean segregation errors. For a large range of parameters, no or only little segregation occurred (0.40.6 range). In particular, this is the case if $c^{(r e p u l)}$ is 0 or if $c^{(\text {rand) }}$ is larger or equal to 3.6. The lowest segregation error occurred for $c^{(\text {rand })} \leq 2$.

We performed further independent simulation runs to sample points over a more focused range of parameter combinations: $c^{(\text {rand })} \in\{0,0.1,0.2,0.3, \ldots, 2\}$ and $c^{(\text {repul })} \in$ $\{0,0.1,0.2,0.3, \ldots, M / 2\}$. For each combination we conducted 100 runs (i.e., 136500 independent simulation runs in total).

Fig. 4 shows the mean segregation errors. Note that in this figure, color levels represent the errors in log-scale. Two values (in white) are out of the considered range; the corresponding segregation errors are thus below $0.5^{10}$. The segregation errors do not change much for repulsion coefficients larger than 2 (if the random coefficient is kept constant at the same time). This is likely caused by function $f$ [see Equation (8)], which limits the overall impact of repulsions in relation to the robot's maximum speed.

We conducted further independent simulation runs in order to choose the best parameter combination (among all parame- 


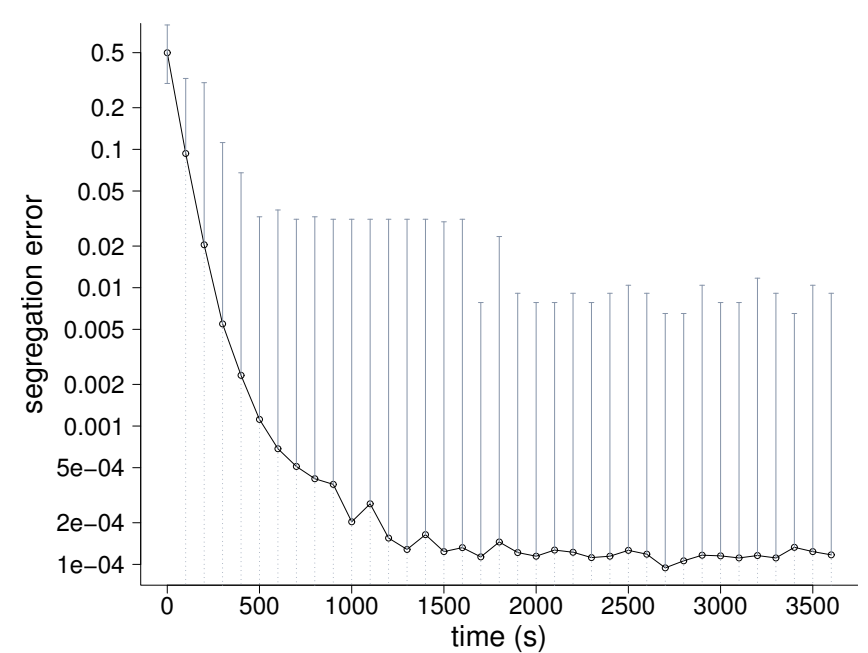

Fig. 5. Mean segregation error with the best found parameter combination, $c^{(\text {rand })}=0.6$ and $c^{(\text {repul })}=0.3$, for different run times $T$ (log-scale, 2000 trials per data point). Arrows stretch respectively to the smallest and biggest observations (dotted arrows indicate that the smallest observation is zero)

ter combinations considered in Fig. 4) . In particular, we used a racing algorithm [18] to identify the parameter combination that is likely to have the lowest expected segregation error (using a $p$-value of 0.05 ). The outcome was $c^{(\text {rand })}=0.6$ and $c^{(\text {repul })}=0.3$. In the following, we analyze the system using this parameter combination in more detail.

\section{B. The influence of time}

In this section, we examine the time dynamics. We consider simulation periods of $T=0,100,200, \ldots, 3600 \mathrm{~s}$. For each $T$, we conducted 2000 simulation runs (i.e., 74000 independent simulation runs in total).

Fig. 5 shows the mean segregation error. At time $T=0$, the mean segregation error is ca. 0.5. This is not surprising as the order of robots is purely random. During the first $600 \mathrm{~s}$, the error rapidly decreases to a value of ca. 0.00069 (note that the plot is in log-scale). As time proceeds, the error further decreases, but only moderately, until it finally settles around a value of ca. 0.00012 (the mean of all observations for $2000 \leq T \leq 3600$ ).

In the following analysis we use a time period of $T=$ $600 \mathrm{~s}$ again.

\section{The influence of errors in the inter-robot perception/ communication}

In this section, we examine the influence of errors in the inter-robot perception and communication on the performance of the system. Let us assume that the disk of robot $i$ intersects with the disk of a nearby robot $j \neq i$. Thus, robot $i$ should get repelled by robot $j$. In this situation, robot $i$ is affected by three types of noise:

1) N1: Robot $i$ detects the intersection with the disk of robot $j$ with probability $u_{1}$ and does not detect the intersection otherwise. The noise level can be controlled from $u_{1}=0(0 \%)$ to $u_{1}=1(100 \%)$.

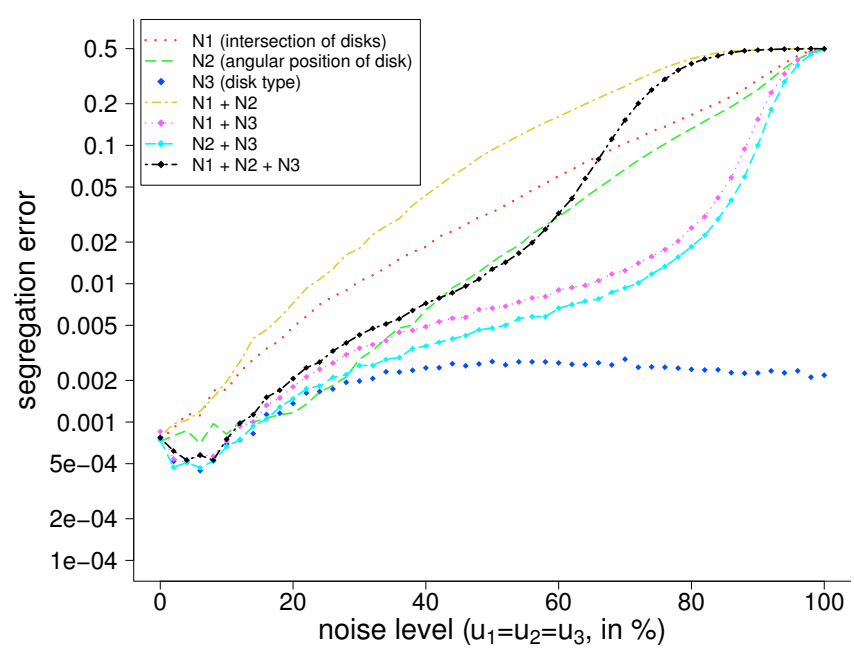

Fig. 6. Mean segregation error with the best found parameter combination, $c^{(\text {rand })}=0.6$ and $c^{(\text {repul })}=0.3$, for different types of noise and for different levels of noise (log-scale, 2000 trials per data point).

2) N2: Provided that robot $i$ detects the intersection with the disk of robot $j$, the angular position of robot $j$ (and its disk), $\gamma_{i, j}$, is subjected to an offset $\xi_{i, j}$. The offset $\xi_{i, j}$ is chosen uniformly from range $\left[-u_{2}, u_{2}\right]$. The noise level can be controlled from $u_{2}=0(0 \%)$ to $u_{2}=\pi(100 \%)$.

3) N3: Provided that robot $i$ detects the intersection with the disk of robot $j$, it detects the disk type of robot $j$ correctly with probability $u_{3}$, and it detects the disk type incorrectly otherwise. In the latter case, we sample uniformly from all incorrect types. The noise level can be controlled from $u_{3}=0(0 \%)$ to $u_{3}=1(100 \%)$.

We consider the seven noise configurations N1, N2, N3, $\{\mathrm{N} 1, \mathrm{~N} 2\},\{\mathrm{N} 1, \mathrm{~N} 3\},\{\mathrm{N} 2, \mathrm{~N} 3\}$, and $\{\mathrm{N} 1, \mathrm{~N} 2, \mathrm{~N} 3\}$ as well as the noise levels $0 \%, 2 \%, 4 \%, \ldots, 100 \%$. We conducted 2000 independent simulation runs for every pair of noise configuration and noise level. That is, we conducted 714000 simulation runs in total.

Fig. 6 shows the mean segregation errors. Overall, the system performs fairly robustly with respect to noise (note that the segregation errors are plotted in log-scale). It is not surprising that the system can not cope with a noise level of $100 \%$ for noise type N1 or N2-in both cases the robot has no information on the angular position of other robots; the resulting ranks are purely random. Consequently, the performance is 0.5 . Interestingly, the system is fairly robust against noise of type N3. Regardless of the level of noise (0-100\%), the mean segregation error was always less than 0.0029. Moreover, noise type N3 cancelled out (at least partially), noise types $\mathrm{N} 1$ and N2. Robots affected by noise types $\mathrm{N} 1$ and $\mathrm{N} 3$ (or by $\mathrm{N} 1, \mathrm{~N} 2$, and N3) performed consistently better, on average, than robots affected only by noise type N1 (or by N1 and N2). Robots affected by noise types N2 and N3 performed better, on average, than robots affected only by noise type $\mathrm{N} 2$, except for noise levels within range $[18,28]$. Recall that noise type N3 concerns the signal, which indicates the disk radii of other robots. A noise level of 


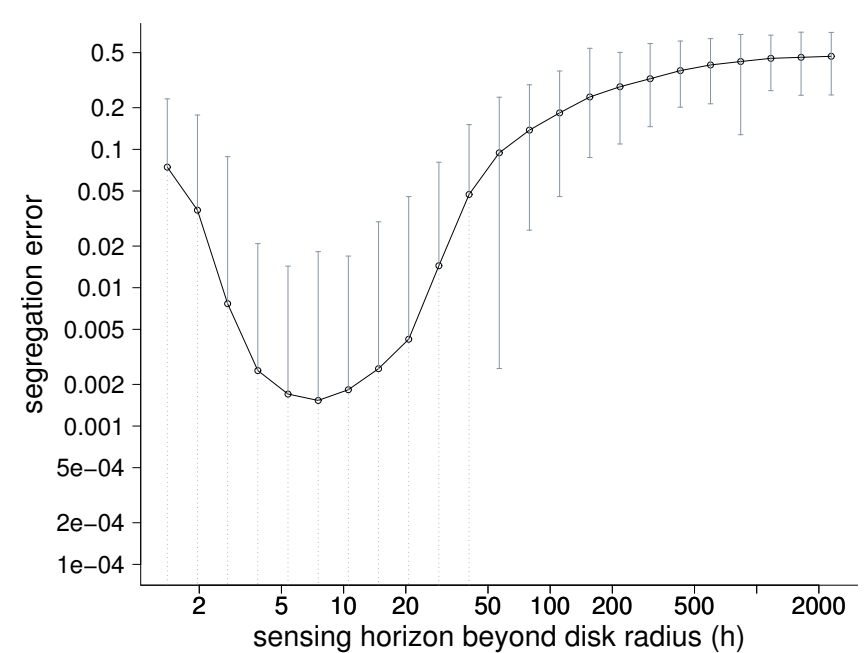

Fig. 7. Mean segregation error with the best found parameter combination, $c^{(\text {rand })}=0.6$ and $c^{(\text {repul })}=0.3$, for a control policy that assumes a unique disk radius $h$ for all other robots (log-scale, 1000 trials per data point). Robot $i$ gets thus repelled by all robots within range $r_{i}+h$. The arrows are explained in the caption of Fig. 5.

$66 \%$ would effectively mean that the robot chooses randomly and uniformly one of the three possible values $\left(r^{(1)}, r^{(2)}\right.$, and $\left.r^{(3)}\right)$. Interestingly, as the noise level further increased to $100 \%$, the mean segregation error slightly decreased. A noise level of $100 \%$ would mean that the robot chooses randomly and uniformly among the two incorrect values. Another counter-intuitive result is that the performance of the swarm slightly increased when the N3 noise level grew from 0 to ca. $6 \%$. A reason for this could be that an occasional misperception of another robot's disk radius can provide the impetus for escaping a spatial arrangement that is only locally optimal, whereas it would not harm to the same extent a spatial arrangement that is globally optimal.

\section{Segregation without communication}

In the previous section, we have seen that a robot does not fundamentally require knowledge of the disk radii of others robots. In this section, we study a simplified version of the control algorithm, which does not rely on communication. In particular, we examine the situation that robot $i, i=$ $1,2,3, \ldots, n$, assumes that all other robots have a constant disk radius $h$. Discrimination is thus based solely on the knowledge of the robot's own disk radius $r^{(i)}$. Robot $i$ is thus repelled by any other robot within sensing range $r^{(i)}+h$. Variable $h$ represents the sensing horizon beyond the robot's own disk radius. We consider sensing horizons $h=1.4^{k}, k=1,2,3, \ldots, 23$ (in $\mathrm{cm}$ ). For each $h$, we conducted 1000 simulation runs (i.e., 23000 independent simulation runs in total). As $h \geq 1.4 \mathrm{~cm}$, the sensing range of each robot is at least twice the radius of its physical body $\left(\min _{i} r_{i}+1.4=7.4=2 \cdot 3.7\right)$.

Fig. 7 shows the mean segregation error (in log-scale). If the sensing horizon is very small, the robots representing the smallest disks do not get repelled by other robots. As a consequence, they form a dense pattern (in the center of the environment), in which other robots can easily get trapped.

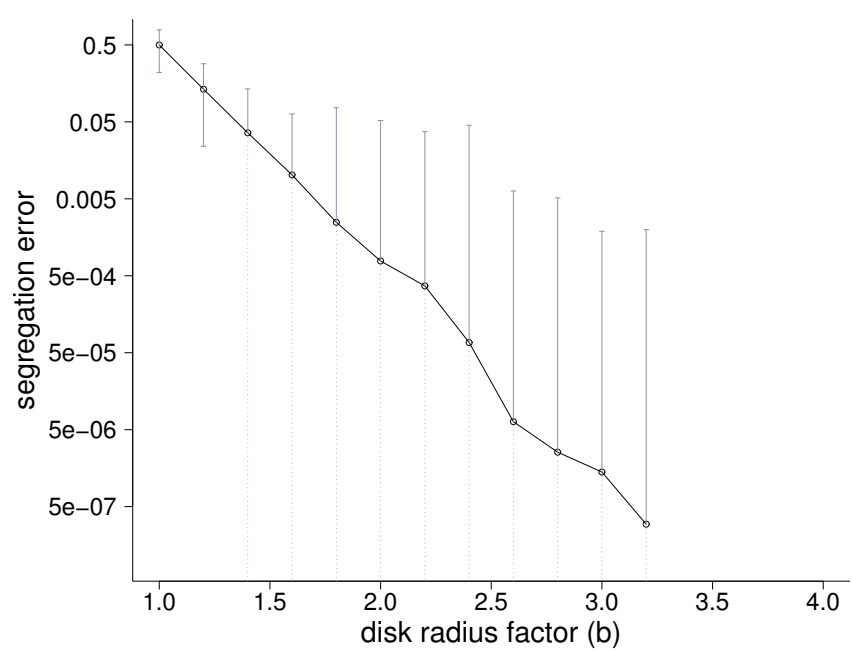

Fig. 8. Mean segregation error with the best found parameter combination, $c^{(\text {rand })}=0.6$ and $c^{(\text {repul })}=0.3$, for different size ratios for the virtual radii (log-scale, 7000 trials per data point). The arrows are explained in the caption of Fig. 5.

If the sensing horizon is very large, the robots spread out in the environment to limit the impact of repulsions. In fact, in their initial configuration, all robots get repelled by all other robots. The robots form large patterns, which certainly takes up valuable time. More importantly, the effect of segregation is limited because the relative differences in the sensing ranges are low.

For an intermediate sensing horizon of about $5-11 \mathrm{~cm}$ (not including the robot's own disk radius), the lowest mean segregation errors were observed: 0.0017, 0.0015, and 0.0018 . For this range of parameters, the maximum error observed was 0.0182 .

\section{E. Error-free segregation by increasing the size ratio}

In this section, we examine the influence of control parameter $b$ - the minimum size ratio between disks of different groups [see Equation (10)]. We consider size ratios $b=$ $1.0,1.2,1.4,1.6, \ldots, 4.0$. For each $b$, we conducted 7000 simulation runs (i.e., 112000 independent simulation runs in total).

Fig. 8 shows the mean segregation error (in log-scale). For $b=1$, all disks had the same size. Consequently, no segregation occurred. The mean error decreased exponentially as control parameter $b$ was increased. For $b=3.4,3.6,3.8$, and 4.0 , the mean error was 0 , that is, the segregation was errorfree at the end of the corresponding 28000 simulation runs. As $b$ increases from 2 to 4 , the curve becomes less smooth (in log-scale). This can be attributed to the decreasing number of runs that still led to an error in segregation: $754(b=2.0)$, $219(b=2.2), 85(b=2.4), 22(b=2.6), 8(b=2.8), 5$ $(b=3.0), 1(b=3.2)$, and 0 thereafter.

It is worth noting that the smallest possible segregation error, $\epsilon$, is bounded and that it decreases at most quadratically with $n$ :

$$
\epsilon \geq \frac{1}{\left(\begin{array}{l}
n \\
2
\end{array}\right)} \geq \frac{1}{n^{2}}
$$




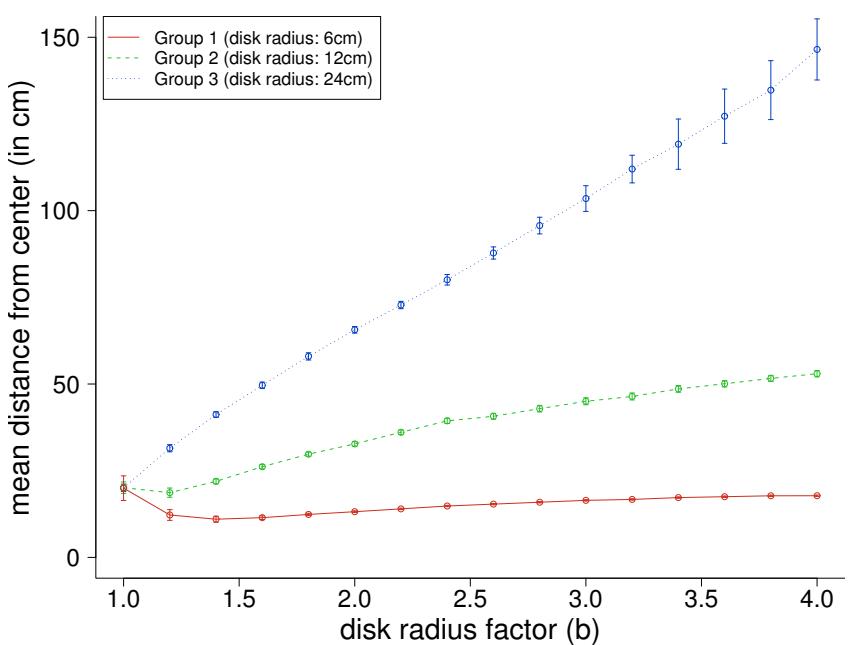

Fig. 9. Mean distance between the disks and the center for groups of different virtual radii (linear scale, 4000 trials per data point). Arrows indicate error bars.

On the other hand, the mean segregation error decreased at an exponential rate. Therefore, by moderately increasing control parameter $b$, one can potentially cope with huge swarms of robots.

To understand how an increase in parameter $b$ makes errorfree segregation possible, we examine the spatial distribution of robots of different groups. Fig. 9 shows the mean distance between the robots and the center (for the different groups). The arrows in this figure indicate error bars (we did not use error bars in the other plots, as the latter were in log-scale and as some of the individual observations were zero). For $b=1$, all disks had the same size. Consequently, no segregation in space occurred. As $b$ increased, one can see that the clusters drifted apart. The standard deviations are small. One can also recognize that groups 2 and 3 are further apart than groups 1 and 2. The reason for this could be that the larger the disk sizes of a group, the less dense the packing [the distribution characterized by Equation (11) assumes that all disks can be packed at density 1].

Further research is necessary to investigate in detail the relationship of error-free segregation and noise. With a setting of $b=4$, a swarm of 50 robots produced error-free segregation in all of 40000 additional simulation runs for a moderate noise level of $2.5 \%$ on all sensors (the actuators were subjected to noise as usual).

\section{PATH TOWARDS IMPLEMENTATION}

We have implemented and tested the control strategy using the physics based simulation toolkit Enki. We are planning to implement the control strategies using the e-puck [15] or swarm-bot [19] robotic platforms. Controllers developed with Enki have already been ported successfully to these robotic platforms (e.g., [20]).

For the e-puck robotic platform, a range \& bearing board is available [21]. For distances less than $600 \mathrm{~cm}$, the board is reported to operate with a maximum error of $39 \mathrm{~cm}$ in range and 27 degrees in bearing. Another option for the e-puck robot would be the omni-directional vision turret [22]. This board is reported to allow robots to detect the signals emitted by any other robot up to a distance of $100 \mathrm{~cm}$.

The robots of the swarm-bot platform have built-in omnidirectional vision and communication systems (based on colored LEDs). These have been extensively used in robotic experimentation [23], [20], [24]

In a real scenario a beacon could be put above the center location, so that all robots can localize the angular position of the center. In a bounded environment, one could exploit the Earth's magnetic field, or its gravity in a slightly inclined environment (both robots have accelerometers).

\section{CONCLUSiON}

We proposed a control algorithm to let mobile robots selforganize into annular structures. The algorithm is inspired by the Brazil nut effect. Using a physics-based computer simulation, we performed a comprehensive quantitative analysis of the performance of a swarm of 50 robots. We showed that the segregation performance is very robust to various types of noise. This led to a simpler version of the algorithm, which does not require communication. We observed that the segregation error decreased exponentially when the size ratio of the particles increased. This allows for error-free segregation provided that the sensing range of the robots is sufficiently large. It is worth noting that these properties concern the formation of annular structures in open space. The extent to which they apply to the formation of other structures in more complex environments, for an example, see Fig. 2, has yet to be determined and is subject to ongoing investigation. Future work will also investigate these properties in more detail (e.g., in larger swarms), as well as test the algorithms (with and without communication) on a physical robotic system.

\section{REFERENCES}

[1] S. Camazine, J.-L. Deneubourg, N. R. Franks, J. Sneyd, G. Theraulaz, and E. Bonabeau, Self-organization in biological systems. Princeton Univ. Press, Princeton, NJ, 2001.

[2] E. Bonabeau, M. Dorigo, and G. Theraulaz, Swarm intelligence: From natural to artificial systems. Oxford Univ. Press, New York, 1999.

[3] D. J. T. Sumpter, "The principles of collective animal behaviour," Phil. Trans. Roy. Soc. B, vol. 361, no. 1465, pp. 5-22, 2006.

[4] S. Garnier, J. Gautrais, and G. Theraulaz, "The biological principles of swarm intelligence," Swarm Intelligence, vol. 1, no. 1, pp. 3-31, 2007

[5] C. W. Reynolds, "Flocks, herds and schools: A distributed behavioral model," in Proc. of the 14th Annual Conf. on Computer Graphics and Interactive Techniques (SIGGRAPH'87). ACM Press, New York, 1987, pp. 25-34.

[6] A. Rosato, K. J. Strandburg, F. Prinz, and R. H. Swendsen, "Why the Brazil nuts are on top: Size segregation of particulate matter by shaking," Phys. Rev. Lett., vol. 58, no. 10, pp. 1038-1040, 1987.

[7] G. Barker and M. Grimson, "The physics of muesli," New Scientist, vol. 126 , no. 1718 , pp. $37-40,1990$.

[8] J. C. Williams and M. I. Khan, "The mixing and segregation of particulate solids of different particle size," Chem. Eng. (London), vol. 269, pp. 19-25, 1973.

[9] C. Melhuish, O. Holland, and S. Hoddell, "Collective sorting and segregation in robots with minimal sensing," in Proc. of the 5th Int. Conf. on Simulation of Adaptive Behavior (SAB'98). MIT Press, Cambridge, MA, 1998, pp. 465-470. 
[10] C. Melhuish, A. B. Sendova-Franks, S. Scholes, I. Horsfield, and F. Welsby, "Ant-inspired sorting by robots: The importance of initial clustering," J. Roy. Soc. Interface, vol. 3, no. 7, pp. 235-242, 2006.

[11] M. Wilson, C. Melhuish, A. B. Sendova-Franks, and S. Scholes, "Algorithms for building annular structures with minimalist robots inspired by brood sorting in ant colonies," Auton. Robot., vol. 17, no. 2-3, pp. 115-136, 2004.

[12] N. R. Franks and A. B. Sendova-Franks, "Brood sorting by ants: Distributing the workload over the work-surface," Behav. Ecol. Sociobiol., vol. 30, no. 2, pp. 109-123, 1992.

[13] S. Magnenat, M. Waibel, and A. Beyeler, "Enki: The fast 2D robot simulator," http://home.gna.org/enki/, 2007.

[14] Cyberbotics, "Webots ${ }^{\mathrm{TM}}$ 6," http://www.cyberbotics.com.

[15] F. Mondada, M. Bonani, X. Raemy, J. Pugh, C. Cianci, A. Klaptocz, S. Magnenat, J.-C. Zufferey, D. Floreano, and A. Martinoli, "The e-puck, a robot designed for education in engineering," in Proc. of the 9th Conf. on Mobile Robots and Competitions (ROBOTICA 2009). IPCB: Instituto Politécnico de Castelo Branco, Castelo Branco, Portugal, 2009, pp. 59-65.

[16] M. Matsumoto and T. Nishimura, "Mersenne twister: A 623dimensionally equidistributed uniform pseudo-random number generator," ACM Trans. Model. Comput. Simul., vol. 8, no. 1, pp. 3-30, 1998.

[17] R. C. Arkin, "Motor schema-based mobile robot navigation," Int. J. Robot. Res., vol. 8, no. 4, pp. 92-112, 1989.

[18] M. Birattari, T. Stützle, L. Paquete, and K. Varrentrapp, "A racing algorithm for configuring metaheuristics," in Proc. of the Genetic and Evolutionary Computation Conf. (GECCO'02). Morgan Kaufmann, San Francisco, 2002, pp. 11-18.

[19] F. Mondada, L. M. Gambardella, D. Floreano, S. Nolfi, J.-L. Deneubourg, and M. Dorigo, "The cooperation of swarm-bots: Physical interactions in collective robotics," IEEE Robot. Automat. Mag., vol. 12, no. 2, pp. 21-28, 2005.

[20] D. Floreano, S. Mitri, S. Magnenat, and L. Keller, "Evolutionary conditions for the emergence of communication in robots," Curr. Biol., vol. 17, no. 6, pp. 514-519, 2007.

[21] A. Gutiérrez, A. Campo, M. Dorigo, D. Amor, L. Magdalena, and F. Monasterio-Huelin, "An open localization and local communication embodied sensor," Sensors, vol. 8, no. 11, pp. 7545-7563, 2008.

[22] D. Floreano, S. Mitri, and J. Hubert, "A robotic platform for studying the evolution of communication," in Evolution of Communication and Language in Embodied Agents, S. Nolfi and M. Mirolli, Eds. Springer Verlag, Berlin, Germany, in press.

[23] E. Tuci, R. Groß, V. Trianni, F. Mondada, M. Bonani, and M. Dorigo, "Cooperation through self-assembly in multi-robot systems," $A C M$ Trans. on Autonomous and Adaptive Systems, vol. 1, no. 2, pp. 115$150,2006$.

[24] R. Groß, S. Nouyan, M. Bonani, F. Mondada, and M. Dorigo, "Division of labour in self-organised groups," in Proc. of the 10th Int. Conf. on Simulation of Adaptive Behavior (SAB 2008), ser. Lect. Notes Comput. Sc., vol. 5040. Springer Verlag, Berlin, Germany, 2008, pp. 426-436. 